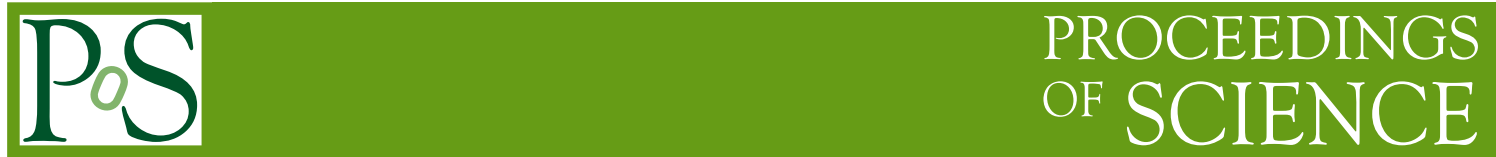

\title{
Top Physics at the Tevatron
}

\author{
Mikhail Arov*† \\ Louisiana Tech University \\ E-mail: arovefnal.gov
}

This paper presents the latest developments in top physics by the D0 and CDF collaborations at the Tevatron Collider in Fermilab using about $3 \mathrm{fb}^{-1}$ of data. The most important one was observation of the electroweak production of single top quarks. Top quark pair production and top mass measurements were also vastly improved. Finally we present searches for new physics beyond the Standard Model, specifically for Charged Higgs bosons and resonant mode top top pair production.

12th International Conference on B-Physics at Hadron Machines - BEAUTY 2009

September 07 - 112009

Heidelberg, Germany

\footnotetext{
* Speaker.

$\dagger^{\dagger}$ For D0 and CDF collaborations.
} 


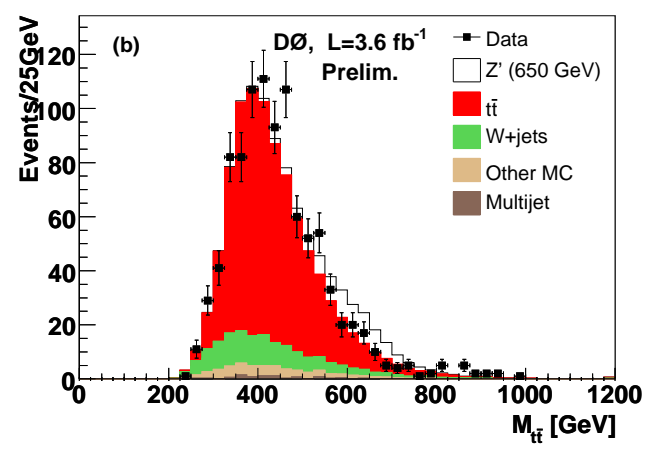

Figure 1: Top quark candidates in the D0 lepton+jets channel [3]. Red is $t \bar{t}$. More then 1000 events are observed by D0 Collaboration.

\section{Introduction}

The top quark is old news. It was discovered almost 15 years ago by two scientific collaborations, D0 [1] and CDF [2]. But even after all these years D0 and CDF are still the only two places where this particle can be observed and studied. This is because D0 and CDF are two main experiments on the Tevatron, the highest energy hadron collider in the world to this day.

But, due to the efforts of only two relatively small teams top physics evolved by leaps and bounds from its humble beginnings in 1995. The top quark was discovered with an observation of only 19 events by CDF and 17 by D0. Today each collaboration has collected over 1000 top events, as can be seen in Fig 1 for instance. In this report we present the latest findings based on over 3 $f b^{-1}$ of data collected.

The top quark is by far the heaviest elementary particle known (172.5 GeV according to the latest measurements) and because of this it can only be produced at the Tevatron, a $2 \mathrm{TeV}$ center of mass proton-antiproton collider. It occupies a unique position among elementary particles with a mass close to the mass of an entire gold atom and $\Gamma \sim 1.4 \mathrm{GeV}>>\Lambda_{Q C D}$.

\section{Top physics overview}

Within the Standard Model of particle physics, the top quark is characterized by only a handful of parameters. The main ones are the top mass and the three CKM matrix elements involving top. The two off-diagonal elements $\left(V_{t d}\right.$ and $\left.V_{t s}\right)$ are very small and can not be measured directly (see [4] for some of the indirect constraint results from $B-\bar{B}$ oscillations). Therefore $V_{t d}$ and $M_{t o p}$ are the most important measurements for the top quark.

But before studying the properties of top quark we need to actually observe its production. The two production modes of the top quark at the Tevatron are top-antitop pairs (Fig. 2) and single top (Fig. 2).

The observed top quark events are used to measure $V_{t d}$ and $M_{t o p}$ and many other interesting properties: spin correlations, charge asymmetry, $W$ helicity etc. 


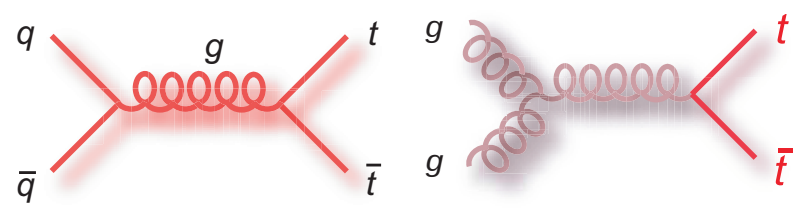

Figure 2: Representative Feynman diagrams for $t \bar{t}$ production at the Tevatron.

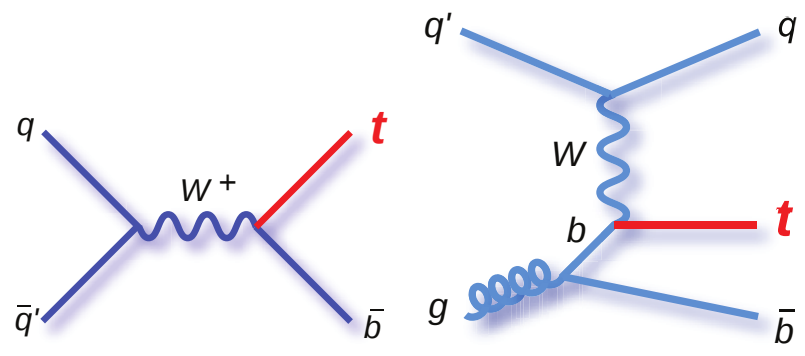

Figure 3: Representative Feynman diagrams for single top production at the Tevatron.

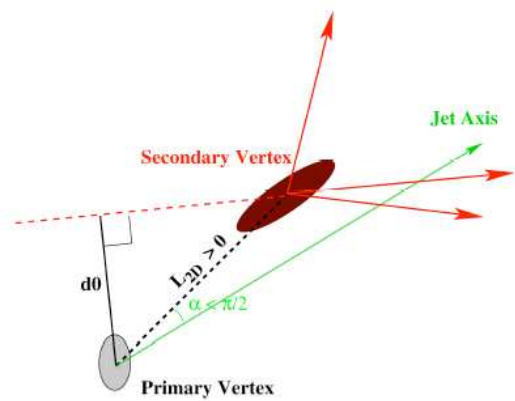

Figure 4: $b$ tagging using the Impact Parameter $d 0$.

\section{3. $b$-tagging}

Since a $b$-quark appears more than $99 \%$ of the time in $t$-quark decays $b$-tagging is the is a very important tool for studying top. There are three effective ways to distinguish $b$-jets from the light quark jets:

- soft lepton tagging (SLT) - $b$-jets can be identified by the presence of an associated muon $(B R(b \rightarrow \mu) \sim 10 \%)$.

- secondary vertex tagging (SVT) - a $b$-jet has a displaced vertex due to the finite lifetime of the heavy quark.

- Impact Parameter (IP) tagging - Similar to SVT but relies on the impact parameters of the tracks contributing to the displaced vertex (see Fig 4).

Each of these has its advantages and disadvantages but the best results are obtained by combining them in one Neural Network discriminant. This improves $b$-tag efficiency by about $30 \%$ 
without sacrificing purity [5].

\section{Top Production}

The main ("golden') channel for top quark production is in the "lepton+jet" final state with Feynman diagram shown in Fig 5(a). The most important background for this channel is $W+$ jets, which has very similar topology (Fig 5(b)). Another crucial background source consists of multi jet QCD events in which one of the jets is misidentified as a lepton (Fig 5(c)).
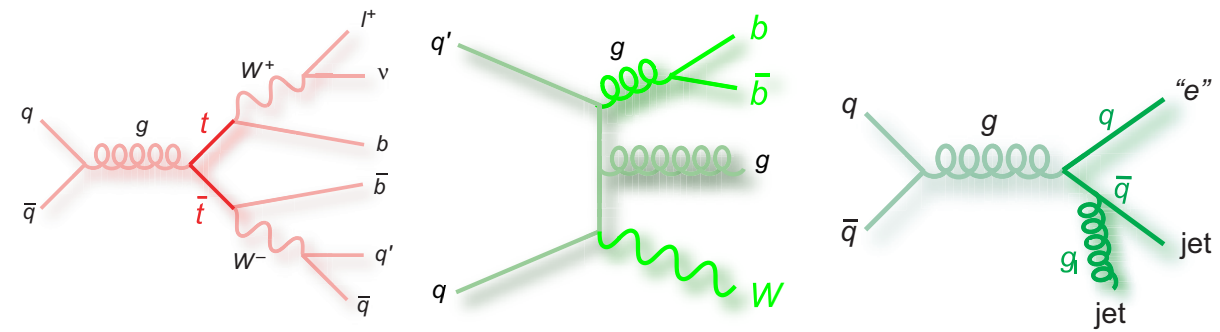

Figure 5: Feynman diagrams for $t \bar{t} \rightarrow l+$ jets (a) and two of its main background sources: $W+$ jets (b) and multi jet QCD (c).

Both CDF and D0 have developed very sophisticated algorithms for reducing these backgrounds and enhancing the signal. The major components are $b$-tagging (described in the previous section) and artificial Neural Networks $[6,7]$.

As a result of applying these techniques to more than $3 \mathrm{fb}^{-1}$ of data, both collaborations managed to measure the $t \bar{t}$ cross section with total uncertainty of about $10 \%$ [8]. This is below the theoretical uncertainty.

\section{Single Top}

While top quark pair production was discovered in 1995, single top discovery was only achieved over a decade later. Part of the reason for this is that single top production has a factor of two lower cross-section than $t \bar{t}$ at the Tevatron. But much more important is the fact that single top appears nearly indistinguishable topologically from its $W+$ jets background. It took careful study of dozens of characteristic variables using some of the most advanced multivariate techniques available today, such as Boosted Decision Trees (BDT) $[9,10]$ to finally make the single top observation.

And it also took 10 years of data taking. Figure 6 demonstrates the latest results from D0 and CDF with both collaborations achieving over $3 \sigma$ significant observation of the single top quark.

\section{Top Mass}

Mass is one of the most important measurable parameters of the top quark. The top-quark mass is also a fundamental parameter in the Standard Model and affects electroweak radiative corrections. It can also be used to indirectly constrain the mass of the still undiscovered Higgs boson [11]. 


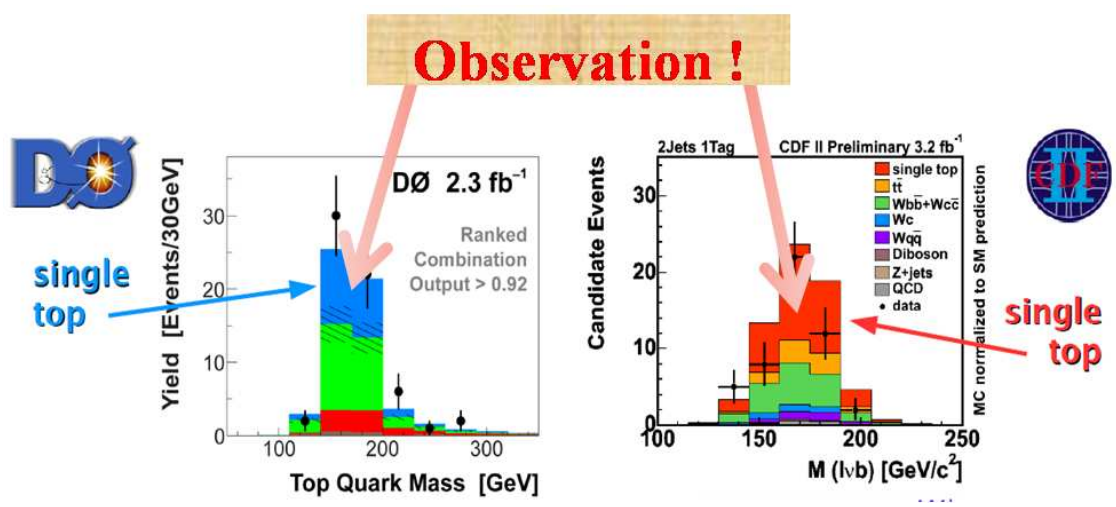

Figure 6: Single top observation. Single top is shown as a blue histogram on the D0 plot and as red on the CDF plot. Three $\sigma$ is achieved in both cases.

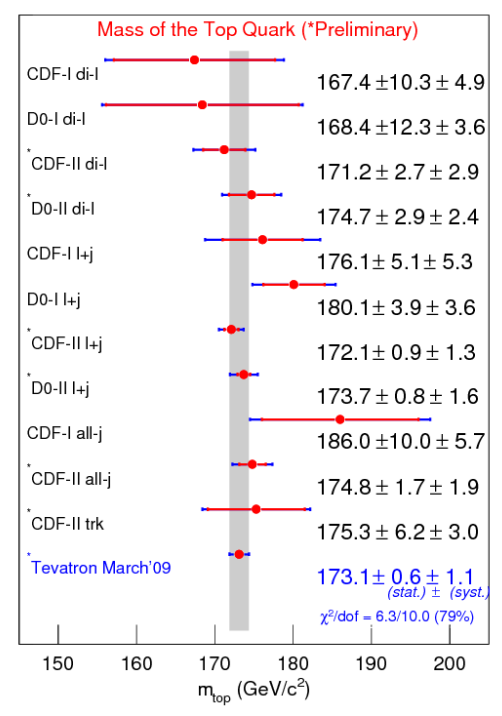

Figure 7: Top mass results from D0 and CDF [14].

There are two principle methods for measuring the top quark mass: the template method and the matrix method. The template method uses MC templates with different masses to fit to top data [12]. The matrix method uses a probability density that is a function of the top mass. The probability density function accounts for the acceptance for the top pair signal, the cross section matrix element and a transfer function, which connects measured jet objects with original quarks [13].

Both methods do an admirable job measuring the top mass, which was measured with less then $1 \%$ uncertainly, as summarized in Figure 7.

\section{Charged Higgs}

As we mentioned in the introduction, the top quark is a powerful tool for new physics searches. One area where the top is indispensable is searches for an extended Higgs sector, in particular the 


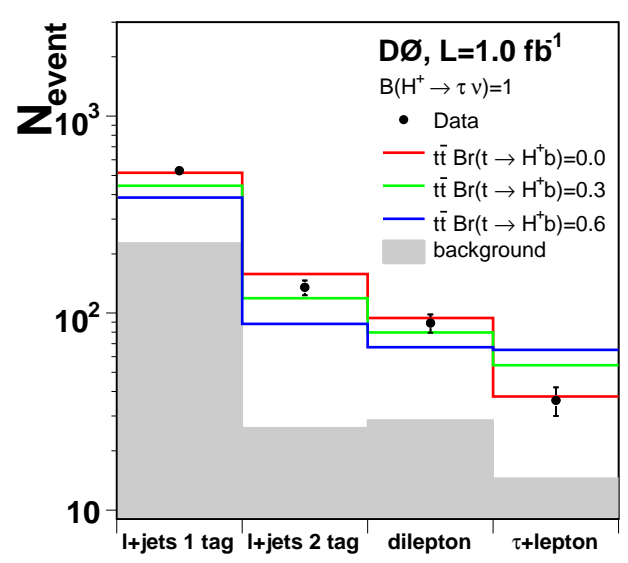

Figure 8: Charged Higgs expected signal at different values of top to $\mathrm{H}^{+}$branching ratio.

Charged Higgs boson $\left(\mathrm{H}^{+}\right)$predicted by MSSM.

In the Standard Model the top quark always decays to a $W$ boson and a $b$ quark. If an $\mathrm{H}^{+}$ exists and is lighter than top then we can have the following scenarios:

$$
\begin{aligned}
& t \bar{t} \rightarrow W^{+} b W^{-} b \\
& t \bar{t} \rightarrow W^{+} b H^{-} b \\
& t \bar{t} \rightarrow H^{+} b W^{-} b \\
& t \bar{t} \rightarrow H^{+} b H^{-} b
\end{aligned}
$$

The Charged Higgs then decays almost exclusively to a $\tau$ lepton. This is very different from the $W$ channels, which respect the flavor universality of the Standard Model. As a result of this, if an $\mathrm{H}^{+}$exists, the event yield of top pair final states that include $\tau$ leptons would increase and decrease for non- $\tau$ final states (Fig. 8).

This allows the Tevatron collaborations to perform Charged Higgs searches. No evidence of $H^{+}$was observed and new limits were set on it [15].

\section{Search for resonant top production}

Another scenario beyond the Standard Model is resonant production of top quark pairs. An example as such production would be a $Z^{\prime}$ decaying to a top-antitop pair (Fig. 9(a)), but any hypothetical heavy boson would produce such an effect. Both collaborations used top pair candidates to look for evidences of such production mechanism and no such evidence was observed so far (Fig. $9(\mathrm{~b}))$.

\section{Conclusions}

This paper only presented a few selected analyses performed at D0 and CDF. Not all important analyses could be included. For a complete overview of the field please refer to [8].

We however demonstrated here two major developments in top quark physics over the past decade: observation of single top and measurement of top quark mass with precision of more 

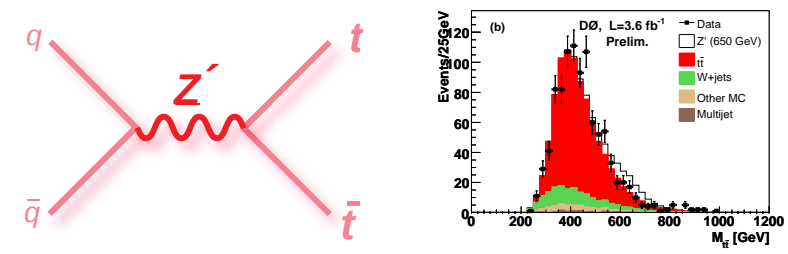

Figure 9: Search for resonant top pair production by D0 [3].

than $1 \%$. These are expected to be greatly improved in future as more data is collected at the Tevatron. Figures 10(a) and 10(b) demonstrate projected development in single top and top mass measurements. We also presented two of the many beyond Standard Model searches made possible using top quark.

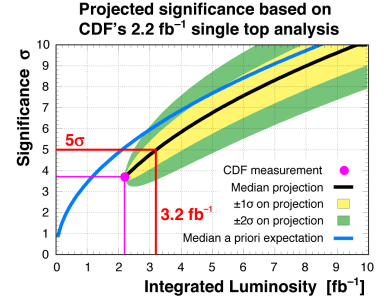

(a) Projected increase in single top significance.

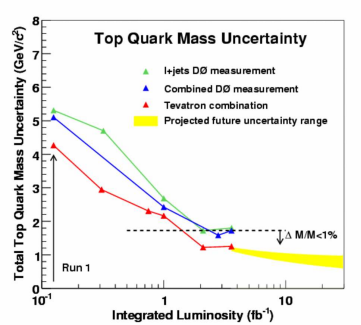

(b) Top quark mass measurement projection.

Figure 10: Projected development of top physics measurements.

\section{References}

[1] S. Abachi et al., (D0 Collaboration), Phys. Rev. Lett. 74, 2632 (1995).

[2] F. Abe et al. (CDF Collaboration), Phys. Rev. Lett. 74, 2626 (1995).

[3] S. Abachi et al., (D0 Collaboration), D0 Conference Note 5828 (2009).

[4] C. Amsler et al., (Particle Data Group), Physics Letters B667, 1 (2008).

[5] T. Scanlon, Ph.D. thesis, FERMILAB-THESIS-2006-43 (2006).

[6] F. Abe et al. (CDF Collaboration), Phys. Rev. D72, 052003 (2005).

[7] F. Abe et al. (CDF Collaboration), CDF Conference Note 9474 (2008).

[8] J. R. Incandela et al., Progress in Particle and Nuclear Physics 63, 239 (2009).

[9] S. Abachi et al., (D0 Collaboration), Phys. Rev. Lett. 103, 092001 (2009).

[10] T. Aalton et al. (CDF Collaboration), Phys. Rev. Lett. 103, 092002 (2009).

[11] J. F. Gunion, H. E. Haber, G. L. Kane and S. Dawson, Higgs Hunter's Guide, Addison-Wesley, Reading, MA (1989).

[12] T. Aalton et al. (CDF Collaboration), CDF Conference Note 10033 (2009).

[13] S. Abachi et al., (D0 Collaboration), D0 Conference Note 5897 (2009). 
[14] Tevatron Electroweak Working Group and CDF Collaboration and D0 Collaboration, FERMILAB-TM-2427-E (2009).

[15] S. Abachi et al., (D0 Collaboration), Phys. Lett. B682, 278-286 (2009)

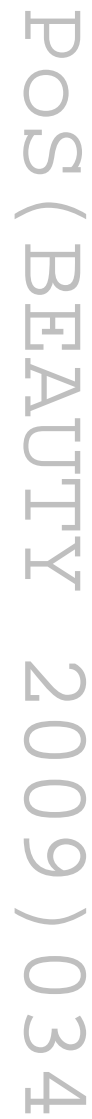

